\title{
MULTIPLE CLASSIFIERS AND GRAPH CUT METHOD FOR SPECTRAL SPATIAL CLASSIFICATION OF HYPERSPECTRAL IMAGE
}

\author{
Bharath Bhushan Damodaran*, Rama Rao Nidamanuri \\ Department of Earth and Space Sciences, Indian Institute of Space Science and Technology, \\ Trivandrum, India - (bharath.11, rao)@iist.ac.in
}

Commission VI, WG VI/4

KEY WORDS: Hyperspectral image classification, Multiple classifier system, Spectral spatial classification, Random subspace method

\begin{abstract}
:
Hyperspectral image contains fine spectral and spatial resolutions for generating accurate land use and land cover maps. Supervised classification is the one of method used to exploit the information from the hyperspectral image. The traditional supervised classification methods could not be able to overcome the limitations of the hyperspectral image. The multiple classifier system (MCS) has the potential to increase the classification accuracy and reliability of the hyperspectral image. However, the MCS extracts only the spectral information from the hyperspectral image and neglects the spatial contextual information. Incorporating spatial contextual information along with spectral information is necessary to obtain smooth classification maps. Our objective of this paper is to design a methodology to fully exploit the spectral and spatial information from the hyperspectral image for land cover classification using MCS and Graph cut (GC) method. The problem is modelled as the energy minimization problem and solved using $\alpha$-expansion based graph cut method. Experiments are conducted with two hyperspectral images and the result shows that the proposed MCS based graph cut method produces good quality classification map.
\end{abstract}

\section{INTRODUCTION}

Hyperspectral image has the potential to highlight the subtle differences between the materials of interest in the hundreds of spectral bands. The exploitation of hyperspectral image is of interest in recent years for many remote sensing applications such as precision agriculture, environmental monitoring, mineral exploration, land cover management, defence and security etc (Briottet et al., 2006, Cetin et al., 2005, Cloutis, 1996). The processing of hyperspectral image often comes with challenges due to its very high dimensionality and redundant information. Each of the hyperspectral image pixel has to be converted into a meaningful information, in accordance with application. The methods of exploring information from the hyperspectral image is still an active area of research.

Among the many methods available in the literature, supervised image classification is the most common used approach for extracting information from the hyperspectral image in the form of classification map. However the supervised classification of hyperspectral image is limited due to the factors such as high dimension, spectral and spatial variability, and limited available ground truth samples (Camps-Valls et al., 2014, Jimenez and Landgrebe, 1998). Different methods have been deployed in literature to overcome the above limitation, such as feature extraction, feature selection methods and non-parametric classifiers such as kernel methods and neural networks etc (Amato et al., 2009, CampsValls and Bruzzone, 2005, Fabio et al., 1997, Gurram and Kwon, 2014, Wang and Chang, 2006). However identifying the optimal classifier for the problem at hand is a challenging task, given the availability of numerous classifier in the literature. Moreover, determining the class, classifier and dimensionality reduction method relationship is necessary to obtain the optimal classification performance (Damodaran and Nidamanuri, 2014a).

In recent years, multiple classifier system (MCS) has evolved as a new classification strategy to merge the advantages of multi-

\footnotetext{
${ }^{*}$ Corresponding author.
}

ple classifier in a single framework to enhance the accuracy and reliability of the classification maps (Damodaran and Nidamanuri, 2014b, Samiappan et al., 2013, Xia et al., 2014). In order to have a successful MCS, the classifiers forming the MCS has to be diverse. Creating the diversity among the base classifiers and combination function are the two major components of the MCS. Apart from the spectral information, hyperspectral images are also rich in the spatial information. Hence incorporating the spatial contextual in the classification framework is essential to achieve the smooth classified maps, otherwise it results in so called salt and pepper noise effect.

The objective of this paper is to design a methodology to exploit the spectral and spatial information from the hyperspectral image for land cover classification using MCS and Graph cut (GC) method. The problem is modelled as the energy minimization problem on the graph of image pixels. The energy is computed as the sum of the data energy term and smoothness term. The data energy term is derived from the MCS and smootheness are modelled using Potts model. The energy minimization problem is solved using alpha expansion method (Boykov et al., 2001). The proposed method has been evaluated on the two airborne hyperspectral images covering range of land cover categories.

The remainder of this paper is as follows: Section 2. describes about the proposed method. Section 3. discusses the experimental results and section 4 . concludes the paper.

\section{METHODOLOGY}

\subsection{Multiple classifier system}

Let $\Psi=\left\{\psi_{1}, \psi_{2}, \ldots, \psi_{L}\right\}$ be the base classifiers forming a MCS, and each classifier $\psi_{l}, l=1,2, \ldots, L$ be a function $\psi_{l}$ : $\chi \rightarrow \Omega$ from an input space $\chi \subseteq R^{n}$ to a set of class labels $\Omega=\left\{\omega_{1}, \omega_{2}, \ldots, \omega_{M}\right\}$ ( $M$ is the number of classes). For any given $\mathbf{x} \in \chi$, classifier $\psi_{l}$ produces a vector of decision values 
$\mathbf{d}=\left[d_{l 1}, d_{l 2}, \ldots, d_{l M}\right]$ and $\mathbf{x}$ is assigned to the class which has the maximum probability (decision) value. The classifiers forming the MCS has to be diverse, in order to enhance the classification accuracy. The Random Subspace Method (RSM) is a popular ensemble generation technique and it has been used to generate the base classifiers in the MCS.

The RSM randomly partitions the hyperspectral image bands into $L$ subsets and each subset contains $\frac{P}{L}$ number of bands, where $P$ denotes the number of bands in the original hyperspectral image. Each subset generated from the RSM is considered as the input data source to the base classifiers $\psi$ in the MCS. The base classifiers can be a different classifiers or same classifier with different parameters. The support vector machine (SVM) has demonstrated its superior capability in classifying the hyperspectral image. Hence in this paper, we have used SVM as the base classifier in the MCS. Both the methods (RSM, and SVM) has the potential to over the small size problem of the hyperspectral image.

The resulting decision function values $(\mathbf{d})$ of the each classifier $\left(\psi_{l}, l=1, \ldots, L\right)$ are arranged as the decision profile matrix (DP) as follows.

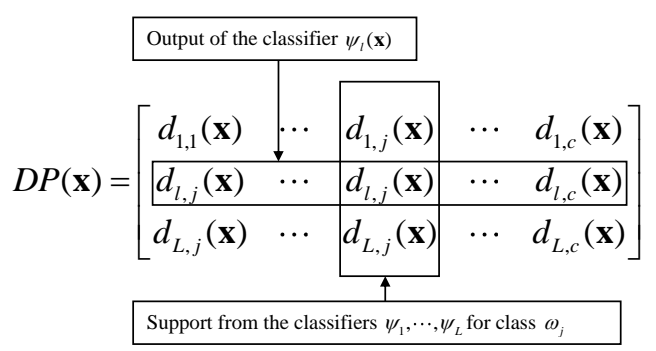

Figure 1: Decision profile matrix of $\mathbf{x}$ from $L$ classifiers.

The $L$ classifiers decision values are combined using Bayesian average combination function (Kittler et al., 1998).

$$
P\left(\frac{\omega_{j}}{\mathbf{x}}\right)=\sum_{l=1}^{L} d_{l, j}(\mathbf{x}), j=1,2, \ldots, M
$$

where $P\left(\frac{\omega_{j}}{x}\right)$ is the posterior probability of the MCS, and the class label for $\mathbf{x}$ is obtained as

$$
\mathbf{x} \in \omega_{m}, m=\underset{j}{\arg \max } P\left(\frac{\omega_{j}}{\mathbf{x}}\right) .
$$

The $P\left(\frac{\omega_{j}}{\mathrm{x}}\right)$ is considered as the pixel wise (spectral) information in the spectral-spatial classification method.

\subsection{Proposed Spectral-Spatial Classification Method}

Markov random field (MRF) model is the powerful mathematical framework to model the spatial contextual information (Moser et al., 2013, Tarabalka et al., 2010). The spatial contextual information of the hyperspectral image is incorporated in the MCS using MRF model. The advantage of applying MRF model in the MCS over the single classifier, is that the MCS results in reliable class posterior probability values. In the effect, this could increase the accuracy than applying the MRF on the single classifier. The problem is formulated as the energy minimization problem as follows

$$
E(\omega)=E_{\text {data }}+E_{\text {smooth }}=\sum_{i=1}^{N} D_{i}\left(\omega_{i}\right)+\sum_{j \in Z} W_{i, j}\left(\omega_{i}, \omega_{j}\right)
$$

where $N$ is the number of pixels in the hyperspectral image, $D_{i}\left(\omega_{i}\right)$ is the potential term which measures the cost of assigning the label $\omega_{i}$ for the pixel $i, Z$ is the spatial neighbourhood pixels of $i^{t h}$ pixel, and $W_{i, j}$ is the interaction term between the adjacent pixels $i$, and $j$.

The data energy term $D_{i}\left(\omega_{i}\right)$ is derived from the estimated class posterior probability for each of the image pixel using MCS. This data energy term is considered as the spectral information of the hyperspectral image.

$$
D_{i}\left(\omega_{i}\right)=-\ln \left(P\left(\frac{\omega_{i}}{\mathbf{x}_{\mathbf{i}}}\right)\right)
$$

The interaction term is expressed by using a Potts model, which penalizes the spatial transitions among neighbouring pixels with different class labels

$$
W_{i, j}\left(\omega_{i}, \omega_{j}\right)=\beta\left(1-\delta\left(\omega_{i}, \omega_{j}\right)\right)
$$

where $\delta($.$) is the Kronecker function \left(\delta\left(\omega_{i}, \omega_{j}\right)=1\right.$ for $\omega_{i}=$ $\omega_{j} ; \delta\left(\omega_{i}, \omega_{j}\right)=0$ for $\left.\omega_{i} \neq \omega_{j}\right)$, and $\beta$ is the positive constant parameter that controls the trade-off between the data energy term and the spatial smoothness term. The equation 3 is solved using a efficient $\alpha$-expansion graph cut based algorithm (Boykov et al., 2001).

\section{EXPERIMENTAL RESULTS AND DISCUSSION}

\subsection{Datasets}

we adopted two benchmark hyperspectral images with different land cover settings (one in urban area and one in agricultural area) captured by two different sensors (ROSIS, AVIRIS) to evaluate the proposed MCS based graph cut method.

ROSIS University: The first hyperspectral dataset was collected over the University of Pavia, Italy by the ROSIS airborne hyperspectral sensor in the framework of HySens project managed by DLR (German national aerospace agency). The ROSIS sensor collects images in 115 spectral bands in the spectral range 0.43 to $0.86 \mathrm{~m}$ with a spatial resolution of $1.3 \mathrm{~m} / \mathrm{pixel}$. After the removal of noisy bands, 103 bands were selected for the experiments. The image contains $610 \times 340$ pixels with nine classes of interest. Figure 2 shows a false color composite (FCC) image and its corresponding ground truth map. The available reference samples in each class are shown in Table 1.

AVIRIS Indian pines: The second hyperspectral image was collected by the AVIRIS sensor over the Indian pines site in the Northwestern Indiana. The AVIRIS sensor collects images in 220 spectral bands in the spectral range 0.43 to $0.86 \mathrm{~m}$ at $20 \mathrm{~m}$ spatial resolution. Twenty water absorption bands were removed, and 200 bands were used for experiments. This image contains 145 x 145 pixels with sixteen classes of interest. Figure 3 shows the FCC image and its corresponding ground truth map. The available reference samples in each class are shown in Table 2.

\subsection{Experimental design}

From the available ground truth samples, we randomly selected 50 samples per class for training, and remaining samples were used for testing. If the total number of available reference samples was lower than 100 samples per class, then $50 \%$ of samples were selected for training, and remaining samples were used as the testing samples. The experimental results were assessed by overall accuracy (OA), average accuracy (AA), and producer accuracy (PA). In order to avoid bias induced by random sampling 


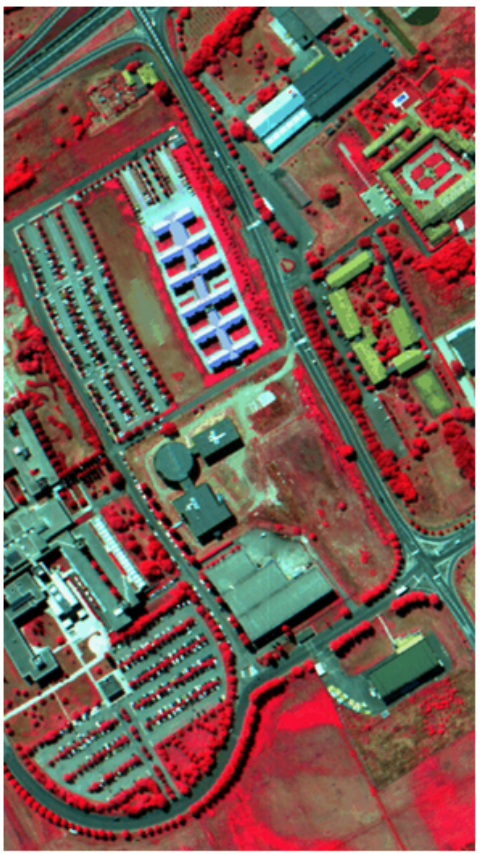

(a)

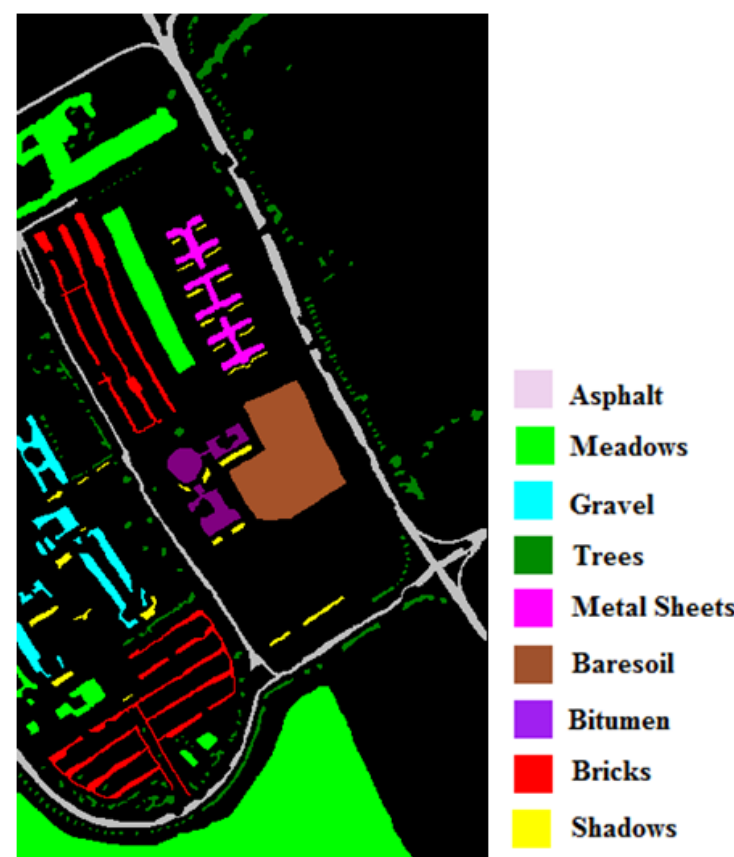

(b)

Figure 2: (a) False color composite of the ROSIS University image (R: $0.8340 \mu \mathrm{m}$ G: $0.6500 \mu \mathrm{m}$ B: $0.5500 \mu m$ ), (b) Ground truth image and its corresponding class labels.

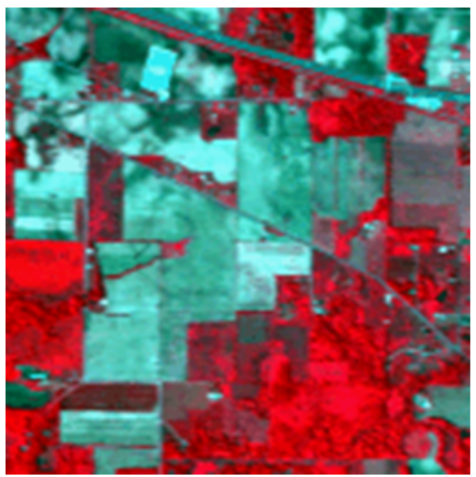

(a)

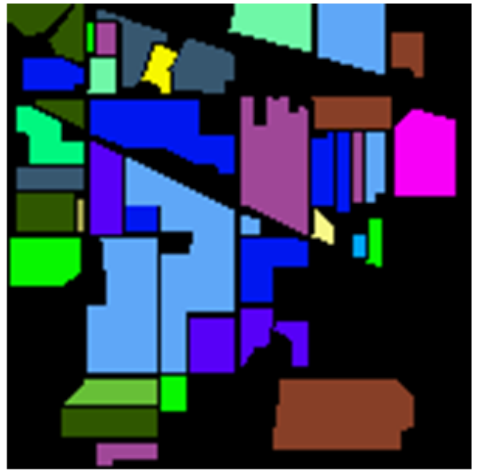

(b)

Figure 3: (a) False color composite of the AVIRIS Indian pines image (R: $0.8314 \mu m$ G: $0.6566 \mu m$ B: $0.5574 \mu m$ ), (b) Ground truth image and its corresponding class labels.

Table 1: Number of reference samples considered for the experiment of University image

\begin{tabular}{ll}
\hline \multicolumn{1}{c}{ Class name } & Reference samples \\
\hline 1. Asphalt & 6631 \\
2. Meadows & 18649 \\
3. Gravel & 2099 \\
4. Trees & 3064 \\
5. Metal sheets & 1345 \\
6. Bare soil & 5029 \\
7. Bitumen & 1330 \\
8. Bricks & 3682 \\
9. Shadows & 947 \\
Total & 42776 \\
\hline
\end{tabular}

Table 2: Number of reference samples considered for the Indian pines image

\begin{tabular}{llll}
\hline \multicolumn{1}{c}{ Class name } & $\begin{array}{c}\text { Reference } \\
\text { samples }\end{array}$ & \multicolumn{1}{c}{ Class name } & $\begin{array}{c}\text { Reference } \\
\text { samples }\end{array}$ \\
\hline 1. Alfalfa & 46 & 9. Oats & 20 \\
2. Corn-notill & 1428 & 10. Soybeans-notill & 972 \\
3. Corn-mintill & 830 & 11. Soybeans-mintill & 2455 \\
4. Corn & 237 & 12. Soybeans-cleantill & 593 \\
5. Hay-windowed & 483 & 13. Wheat & 205 \\
6. Grass/Trees & 730 & 14. Woods & 1265 \\
7. Grass/pasture-mowed & 28 & 15.Bldg & 386 \\
8. Grass/pasture & 478 & 16. Stone-steel towers & 93 \\
Total & & & 10249 \\
\hline
\end{tabular}


of the training samples, ten independent Monte Carlo runs are performed and the accuracies (OA, AA, PA) are averaged over the ten runs.

RSM partitions the hyperspectral image into five random subspaces. In each of these RSM, multiclass pair-wise probabilistic SVM classification with the Gaussian radial basis function (RBF) kernel was performed The SVM parameters in all our experiments were automatically tuned with $C=2^{\alpha}, \quad \alpha=$ $\{-5,-4,, \cdots, 15\}$ and $\gamma=2^{\beta}, \beta=\{-15,-13, \cdots, 3\}(C$ is the cost function and $\gamma$ is the width of the radial basis function) through five-fold cross validation strategy of the training samples. The proposed method are compared with the state-of-the-art pixel wise classification methods such as SVM (fullband SVM), single best classifier, and MCS and with spectral-spatial classification methods such as SVM-GC, and single best classifier-GC (SBC$\mathrm{GC}$ ). The constant parameter $\beta$ in equation 2 is tuned from 0 to 3 manually, and the classification results with the best value of $\beta$ is reported.

\subsection{Experimental Results and Discussion}

3.3.1 MCS results Figure 4 shows the overall accuracy of the base classifiers and MCS for the ROSIS University, and AVIRIS Indian Pines hyperspectral images. The classifier which produces the maximum overall accuracy is known as single best classifier, and this is used as a benchmark to compare the MCS classification results. From the figure 4, it is evident that RSM has produced diverse classification results. The maximum overall accuracy (single best classifier) produced by the RSM is $82.1 \%$ and $74.7 \%$ for the ROSIS University and AVIRIS Indian Pines hyperspectral image respectively.

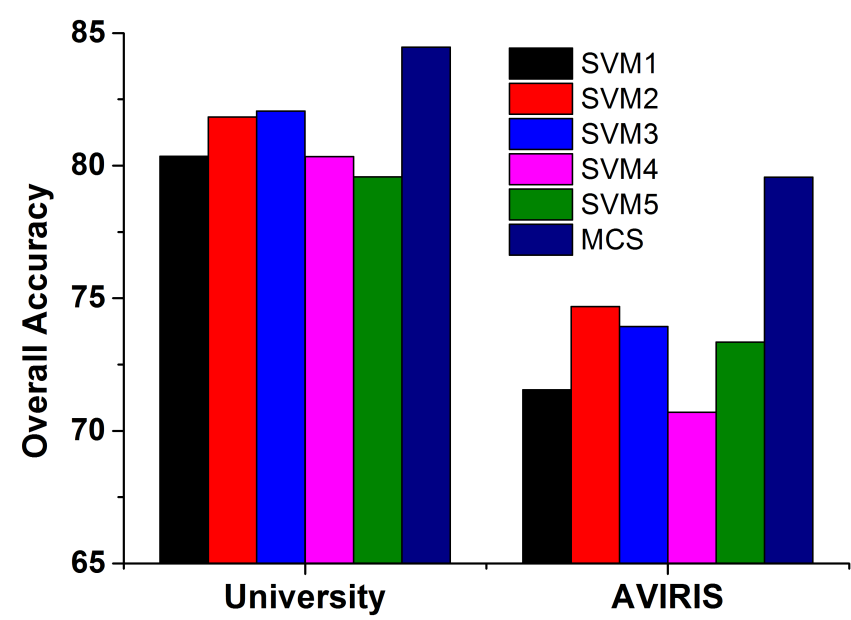

Figure 4: Overall accuracy of the base classifiers in the MCS and the combination function of the MCS (MCS accuracy) for the ROSIS University and AVIRIS Indian Pines hyperspectral images

When the base classifiers decision function values are combined by the Bayesian average combination function, the classification accuracy are increased significantly. For instance, there is $2.5 \%$, and 5\% increase in overall accuracy for the ROSIS University and AVIRIS Indian Pine hyperspectral images respectively. This concludes that the estimated class posterior probability by the MCS are better than the single best classifier. Though the accuracy has been increased, MCS are not able to produce good quality of classification maps.

3.3.2 Results of MCS based graph cut method When the spatial contextual information is incorporated using Markov random field model along with estimated class posterior probability from the MCS, the classification accuracy has increased significantly. The classification accuracy of the MCS based graph cut method is shown in Table 3. The proposed MCS based graph cut method has achieved $12 \%, 11 \%$ increase in overall accuracy over the pixel wise classification (MCS) results. In order to highlight the potential of the proposed method, it is compared with the state-of-the-art pixel wise and spectral-spatial classification methods (see Table 3). When compared with the pixel wise classification methods, the proposed method has yielded an increase about $12-14 \%$ accuracy points for the ROSIS University image and $11-16 \%$ accuracy points for the AVIRIS Indian Pines Hyperspectral images respectively.

Table 3: Overall classification accuracy of our proposed method and comparison with the state-of-the-art pixel wise and spectralspatial classification methods. The classification accuracy is computed over the ten runs and the average accuracy is reported (50 samples are randomly chosen for training and remaining samples are used for testing)

\begin{tabular}{lcccccc}
\hline Image & $\begin{array}{c}\text { Proposed } \\
\text { method }\end{array}$ & SVM & SBC & MCS & $\begin{array}{c}\text { SVM+ } \\
\text { GC }\end{array}$ & $\begin{array}{c}\text { SBC+ } \\
\text { GC }\end{array}$ \\
\hline University & 96.3 & 83.8 & 82.05 & 84.47 & 94.7 & 95.2 \\
AVIRIS & 90.2 & 73.5 & 74.6 & 79.5 & 87.2 & 88.1 \\
\hline
\end{tabular}

When the proposed method is compared with the spectral-spatial classification approaches, the proposed method has yielded 2$3 \%$ improvement in overall accuracy for both the hyperspectral images. Further, kappa statistical significance test confirms that the classification results are statistically significant at $95 \%$ confidence interval. The classified images of the proposed method is shown in Figure 5 and 6. From the figure 5 and 6, it is evident that the salt and pepper noise effect is visible in the pixel wise classified image, where as the classified image in the proposed method is very smooth. This shows that the proposed method is capable to produce good quality classification maps for remote sensing applications.

Table 4: The computational time (in sec) of MCS, our proposed method and other existing methods. The computation time includes cross validation time, training time, testing time and $\alpha$ expansion based graph cut method. The computation has performed on a desktop computer with Intel i3 processor, $3.2 \mathrm{GHz}$, 3 GB RAM and 64 bit operating system

\begin{tabular}{lccc|ccc}
\hline \multirow{2}{*}{ Image } & \multicolumn{2}{c}{ Pixel wise method } & \multicolumn{2}{c}{ Spectral-Spatial method } \\
\cline { 2 - 7 } & SVM & SBC & MCS & $\begin{array}{c}\text { SVM- } \\
\text { GC }\end{array}$ & $\begin{array}{c}\text { SBC- } \\
\text { GC }\end{array}$ & $\begin{array}{c}\text { Proposed } \\
\text { method }\end{array}$ \\
\hline University & 18 & 10 & 11 & 996 & 980 & 981 \\
AVIRIS & 103 & 30 & 31 & 120 & 48 & 47 \\
\hline
\end{tabular}

3.3.3 Computational time complexity Table 4 shows the computational time of the pixel wise classification method, spectral and spatial classification method. From the table 3 and 4, it is obvious that the MCS has outperformed full band SVM classifier in overall accuracy and computational time. It is due to the fact that the base classifiers in the MCS are independent of each other and it can be run parallel. Moreover, the dimension of the input data sources in the MCS is reduced compared to the original hyperspectral image. For instance, the input data dimension in the MCS is about 20 and whereas in the full band SVM classifier is 103 for the ROSIS University hyperspectral image. The computational time of the spectral-spatial classification is high due to computation of the smoothness term. The computation of the smoothness term grows with the number of pixels in the image. Once the smoothness term is computed, the computation of the 


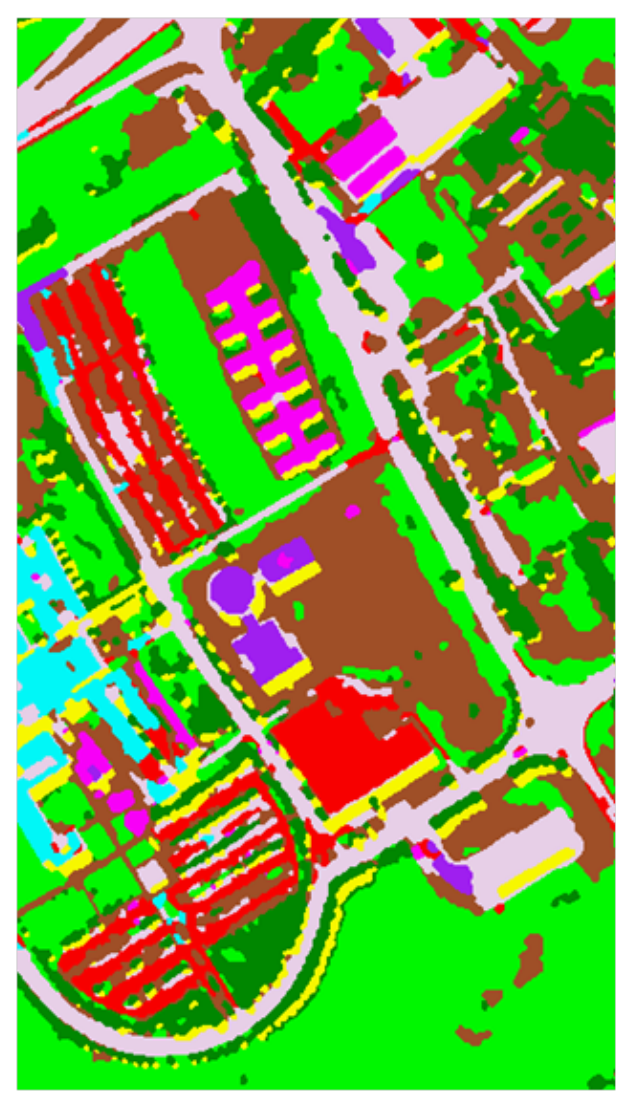

(a)

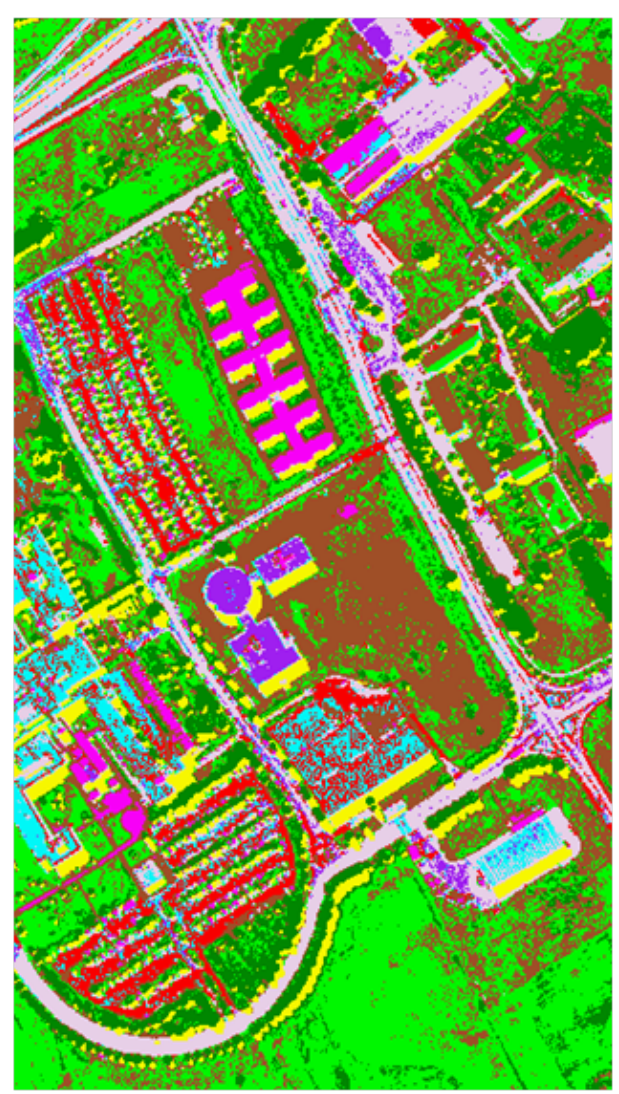

(b)

\section{Asphalt \\ Meadows \\ Gravel \\ Trees}

Metal Sheets

Baresoil

Bitumen

Bricks

Shadows

Figure 5: Classified images of ROSIS University hyperspectral image (a) proposed method (b) SVM (c) corresponding class labels

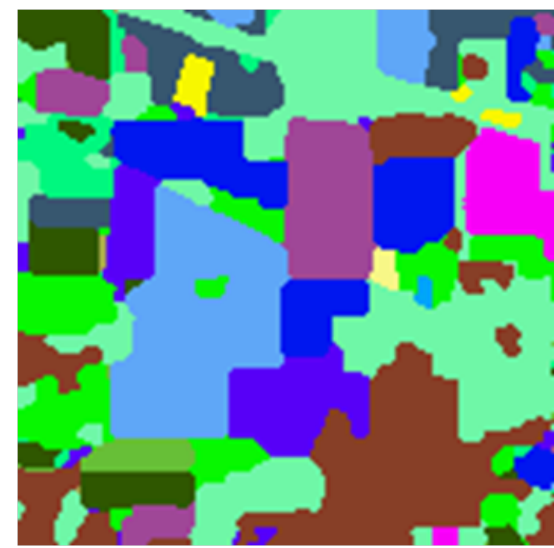

(a)

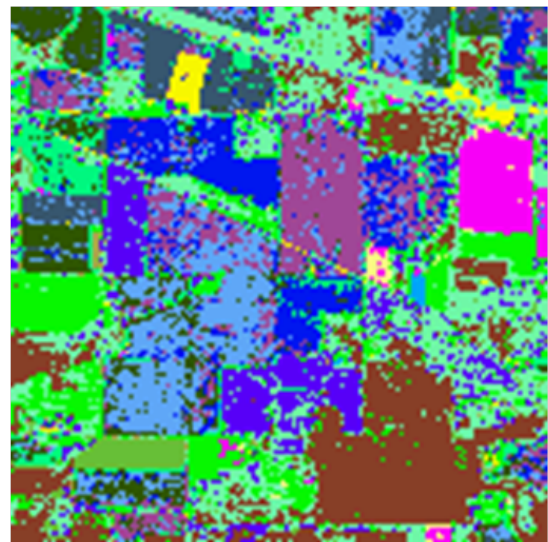

(b)

\begin{tabular}{|l|l|}
\multicolumn{1}{|l|}{ Alfalfa } & Soybeans-notill \\
\hline Corn-notill & Soybeans-mintill \\
\hline Corn-mintill & Soybeans-cleantill \\
\hline Corn & Wheat \\
\hline Hay-windowed & Woods \\
\hline Grass/trees & Bldg \\
\hline Grass/pasture-mowed & Stone-steel towers \\
\hline Grass/pasture & Oats \\
\hline
\end{tabular}

(c)

Figure 6: : Classified images of AVIRIS Indian Pines hyperspectral image (a) proposed method (b) SVM (c) corresponding class labels

$\alpha$-expansion based graph cut method is very fast and converges to global solution.

\section{CONCLUSION}

In this paper, we presented the MCS based graph cut method to exploit the spectral and spatial information from the hyperspectral image. The spectral information is extracted from the MCS and the spatial contextual information is incorporated using MRF model. The experimental results with two airborne hyperspectral image shows that the proposed MCS based graph cut method yields accurate classification over the state-of-the-art methods.
Further, the experiments are conducted with few training samples per class. The proposed method has the potential to produce the high quality classification map for land use/land cover applications.

\section{ACKNOWLEDGEMENTS}

We gratefully acknowledge Prof. Paolo Gamba, Department of Electronics, University of Pavia, Italy for providing us with ROSIS hyperspectral images and ground truth map used in this study. 


\section{REFERENCES}

Amato, U., Cavalli, R. M., Palombo, A., Pignatti, S. and Santini, F., 2009. Experimental Approach to the Selection of the Components in the Minimum Noise Fraction. IEEE Transactions on Geoscience and Remote Sensing 47(1), pp. 153-160.

Boykov, Y., Veksler, O. and Zabih, R., 2001. Fast approximate energy minimization via graph cuts. IEEE Transactions on Pattern Analysis and Machine Intelligence 23(11), pp. 1222-1239.

Briottet, X., Boucher, Y., Dimmeler, A., Malaplate, A., Cini, A., Diani, M., Bekman, H., Schwering, P., Skauli, T., Kasen, I., Renhorn, I., Klasén, L., Gilmore, M. and Oxford, D., 2006. Military applications of hyperspectral imagery. In: W. R. Watkins and D. Clement (eds), Proc. SPIE 6239, Targets and Backgrounds XII: Characterization and Representation, International Society for Optics and Photonics, pp. 62390B-62390B-8.

Camps-Valls, G. and Bruzzone, L., 2005. Kernel-based methods for hyperspectral image classification. IEEE Transactions on Geoscience and Remote Sensing 43(6), pp. 1351-1362.

Camps-Valls, G., Tuia, D., Bruzzone, L. and Atli Benediktsson, J., 2014. Advances in Hyperspectral Image Classification: Earth Monitoring with Statistical Learning Methods. IEEE Signal Processing Magazine 31(1), pp. 45-54.

Cetin, H., Pafford, J. and Mueller, T., 2005. Precision agriculture using hyperspectral remote sensing and GIS. In: Proceedings of 2nd International Conference on Recent Advances in Space Technologies, 2005. RAST 2005., IEEE, pp. 70-77.

Cloutis, E. A., 1996. Review Article Hyperspectral geological remote sensing: evaluation of analytical techniques. International Journal of Remote Sensing 17(12), pp. 2215-2242.

Damodaran, B. B. and Nidamanuri, R. R., 2014a. Assessment of the impact of dimensionality reduction methods on information classes and classifiers for hyperspectral image classification by multiple classifier system. Advances in Space Research 53(12), pp. $1720-1734$.

Damodaran, B. B. and Nidamanuri, R. R., 2014b. Dynamic Linear Classifier System for Hyperspectral Image Classification for Land Cover Mapping. IEEE Journal of Selected Topics in Applied Earth Observations and Remote Sensing 7(6), pp. 20802093.

Fabio, R., Giacinto, G. and Vernazza, G., 1997. Comparison and Combination of Statistical and Neural Network Algorithms for Remote-Sensing. In: Neurocomputation in Remote Sensing Data Analysis, Advances in Spatial Science Series, Springer-Verlag, pp. 117-124.

Gurram, P. and Kwon, H., 2014. Coalition game theory based feature subset selection for hyperspectral image classification. In: 2014 IEEE International Geoscience and Remote Sensing Symposium (IGARSS), pp. 3446-3449.

Jimenez, L. and Landgrebe, D., 1998. Supervised classification in high-dimensional space: geometrical, statistical, and asymptotical properties of multivariate data. IEEE Transactions on Systems, Man and Cybernetics, Part C (Applications and Reviews) 28(1), pp. 39-54.

Kittler, J., Hatef, M., Duin, R. and Matas, J., 1998. On combining classifiers. IEEE Transactions on Pattern Analysis and Machine Intelligence 20(3), pp. 226-239.

Moser, G., Serpico, S. B. and Benediktsson, J. A., 2013. LandCover Mapping by Markov Modeling of SpatialContextual Information in Very-High-Resolution Remote Sensing Images. Proceedings of the IEEE 101(3), pp. 631-651.
Samiappan, S., Prasad, S. and Bruce, L. M., 2013. Non-Uniform Random Feature Selection and Kernel Density Scoring With SVM Based Ensemble Classification for Hyperspectral Image Analysis. IEEE Journal of Selected Topics in Applied Earth Observations and Remote Sensing 6(2), pp. 792-800.

Tarabalka, Y., Fauvel, M., Chanussot, J. and Benediktsson, J. A., 2010. SVM- and MRF-Based Method for Accurate Classification of Hyperspectral Images. IEEE Geoscience and Remote Sensing Letters 7(4), pp. 736-740.

Wang, J. and Chang, C.-i., 2006. Independent Component Analysis-Based Dimensionality Reduction With Applications in Hyperspectral Image Analysis. IEEE Transactions on Geoscience and Remote Sensing 44(6), pp. 1586-1600.

Xia, J., Du, P., He, X. and Chanussot, J., 2014. Hyperspectral Remote Sensing Image Classification Based on Rotation Forest. IEEE Geoscience and Remote Sensing Letters 11(1), pp. 239243. 